Review Article

\title{
Post dural punct: Headache
}

\section{Proceeding}

PDPH can occur both in spinal and epidural due to CSF leakage.

\section{Pathio Physiology of PDPH}

\section{i. CSF leakage.}

PDPH occurs when CSF leakage $>$ CSF Production

ii. Cerebrovasodilation.

Body attempts to maintain homepstasis within cranium.

iii. CSF lead to compensatory Cerebrovasodilation and headache.

iv. Menigeal Irritation.

v. Pnemocephalus.

Accidental intrathecal injection of air during epidural may cause relatively sudden onset of headache.

\section{Risk factors of PDPH}

\section{A. Patient factors:}

a. Gender_females at higher risk.

b. Age_younger pts at higher risk.

B. Needle characteristics:

a. Size larger needle size increases loss of CSF and incidence of PDPH.

b. Shape sharp cutting-edge beveled needle has increased

c. Incidence of PDPH when compared with pencil-point needles.

\section{Control risk factors of PDPH}

\section{Procedure factors:}

a. Needle should be inserted "parallel" to dural fibers. Usually, dural fibers run longitudinally.

b. Paramedian versus midline approach: paramedian may have decreased incidence of PDPH ("flap" rather than a "tin-lid" opening).

c. Mulitiple punctures increase risk of PDPH because of increased loss of CSF.

\section{Clinical assessment of PDPH}
a. Occipto frontal headache.
b. Aggravate sitting position.
c. Patient comfortable supine position.
d. PDPH usually appears 1 to 2 days after dural puncture and lasts (if untreated) for upto 7 days in most cases.
e. Other associated symptoms (not reliable).

Volume 7 Issue 3 - 2017

Lt Col FaizAhmed

Department of Medicine, India

Correspondence: Lt Col Faiz Ahmed, Department of Medicine, Armed Forces Medical College, Pune, India, Tel 9988677867,Email drfaizahmed@gmail.com

Received: October 21, 2016 | Published: March 01, 2017 f. Photphobia, nausea, vomiting, auditory disturbances.

g. Cranial nerve involvement (diplopia).

\section{Treatment of PDPH}

1. Conservative

a. Analgesic + Rehydration and caffine (i/v or oral)

b. Soft diet.

c. Caffenie provides transient relief via vasoconstriction.

2. Invasive.

a. Epidural blood patch (gold standard).

b. Success rate is $70 \%$ to $90 \%$.

c. Two operators required.

d. One takes 15-20 $\mathrm{ml}$ of blood from patient.

Other injects this blood in epidural space at same level or one level below after starile technique. ${ }^{1-3}$

\section{Common tip designs for spinal needles}

Quincke
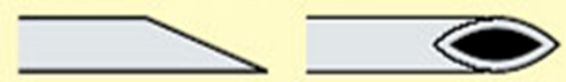

Whitacre
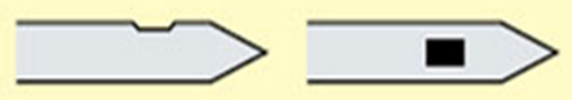

Sprotte
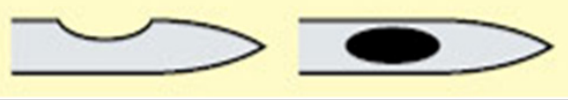

\section{Conflicts of interest}

There is no conflict of interest. 


\section{Acknowledgements}

None.

\section{Funding}

None.

\section{References}

1. Johns Hopkins Anesthesiology Book.

2. MorganText Book.

3. Aitkenheads Text Book. 